\title{
The Cut-Off Values of Anthropometric Indices for Identifying Subjects at Risk for Metabolic Syndrome in Iranian Elderly Men
}

\author{
Mojgan Gharipour, ${ }^{1}$ Masoumeh Sadeghi, ${ }^{2}$ Minoo Dianatkhah, ${ }^{3}$ Shirin Bidmeshgi, \\ Alireza Ahmadi, ${ }^{5}$ Marzieh Tahri, ${ }^{5}$ and Nizal Sarrafzadegan ${ }^{5}$ \\ ${ }^{1}$ Metabolic Syndrome Department, Isfahan Cardiovascular Research Center, Isfahan Cardiovascular Research Institute, \\ Isfahan University of Medical Sciences, Isfahan, Iran \\ ${ }^{2}$ Cardiac Rehabilitation Research Center, Isfahan Cardiovascular Research Institute (WHO Collaborating Center), \\ Isfahan University of Medical Sciences, Isfahan, Iran \\ ${ }^{3}$ Interventional Cardiology Research Center, Isfahan Cardiovascular Research Institute, Isfahan University of Medical Sciences, \\ Isfahan, Iran \\ ${ }^{4}$ Hypertension Research Center, Isfahan Cardiovascular Research Institute, Isfahan University of Medical Sciences, Isfahan, Iran \\ ${ }^{5}$ Isfahan Cardiovascular Research Center, Isfahan Cardiovascular Research Institute, Isfahan University of Medical Sciences, \\ Isfahan, Iran
}

Correspondence should be addressed to Masoumeh Sadeghi; sadeghimasoumeh@gmail.com

Received 28 September 2013; Accepted 27 January 2014; Published 23 March 2014

Academic Editor: Francesco Saverio Papadia

Copyright (C) 2014 Mojgan Gharipour et al. This is an open access article distributed under the Creative Commons Attribution License, which permits unrestricted use, distribution, and reproduction in any medium, provided the original work is properly cited.

\begin{abstract}
Aim. This study aimed to investigate which anthropometric indices could be a better predictor of metabolic syndrome (MetS) and the cut-off points for these surrogates to appropriately differentiate MetS in the Iranian elderly. Method. The present crosssectional study was conducted on a sample of Isfahan Healthy Heart Program (IHHP). MetS was defined according to Third Adult Treatment Panel (ATPIII). In total, 206 elderly subjects with MetS criteria were selected. Anthropometric indices were measured and plotted using receiver operating characteristic (ROC) curves. Results. WC followed by WHtR yielded the highest area under the curve (AUC) $(0.683$; 95\% CI $0.606-0.761$ and $0.680 ; 95 \%$ CI $0.602-0.758$, resp.) for MetS. WC at a cut of $94.5 \mathrm{~cm}$ resulted in the highest Youden index with sensitivity $64 \%$ and $68 \%$ specificity to predict the presence of $\geq 2$ metabolic risk factors. BMI had the lowest sensitivity and specificity for MetS and MetS components. WC has the best ability to detect MetS which followed by WHtR and BMI had a lower discriminating value comparatively. Conclusion. WC is the best predictor for predicting the presence of $\geq 2$ metabolic risk factors among Iranian elderly population and the best value of WC is $94.5 \mathrm{~cm}$. This cut-off values of WC should be advocated and used in Iranian men until larger cross-sectional studies show different results.
\end{abstract}

\section{Introduction}

Obesity increases the risk of cardiovascular disease in adults, has been strongly associated with insulin resistance in nondiabetic persons and in individuals with type 2 diabetes $[1,2]$, and is considered as prerequisite for the diagnosis of metabolic syndrome (MetS). Central obesity is defined using ethnicity-specific cut-off point of waist circumference (WC) [3]. In this regard, previous studies recommended the best cut-off points of WC values for prevention and control of cardiovascular disease in adults and children $[4,5]$.
Definition of obesity differs among various ethnics; therefore, many regional studies are conducted to find the best cut-off points for obesity [6]. The values of anthropometric indices are varied in different age groups with respect to race; for instance, the cut-off points of WC in studies in Oman, Iraq, and Korea were $80 \mathrm{~cm}, 97 \mathrm{~cm}$, and $90 \mathrm{~cm}$, respectively, in men and $84.5 \mathrm{~cm}, 99 \mathrm{~cm}$, and $85 \mathrm{~cm}$, respectively, in women [7]. Numerous national studies determined cut-off points for WC, WHR, and BMI among Iranian adolescents and adults [8-10]. For example, Iranian Multi-Centric Osteoporosis Studies (IMOS) [8] determined of WC in five major cities 
of Iran. Considering the dramatic changes in fat distribution and its function throughout life [11] and according to this fact these changes can have important consequences on the profile of risk factors for developing MetS [12]. Our previous study which was done to determine the prevalence of different phenotypes in various age groups demonstrated that current cut-off points for WC are not appropriate for distinguishing subjects at risk of developing MetS specially among Iranian elderly men [13]. We found that the prevalence of MetS decreased sharply in men above 65 years old, which is related to low prevalence of central obesity in this group. So this study is designed to determine appreciate cut-off points for anthropometric indices in the Iranian elderly especially in men.

\section{Material and Methods}

2.1. Study Population. Isfahan Healthy Heart Program is a comprehensive integrated community-based action-oriented study with a reference community which has been conducted by the Isfahan Cardiovascular Research Institute since 2000 and completed in 2007 [14, 15]. A random independent sample of adults was selected by multistage cluster sampling. The effect of confounding has been addressed by using random, stratified household sampling based on age and sex groups. The participants were more than 19 years old. The samples underwent a 30-minute interview by well-trained examiners to complete validated questionnaires containing questions on demography, socioeconomic status, smoking behavior, physical activity, nutritional habits, and other risk profiles. Informed consent was obtained from all subjects prior to their participation in this study, which was approved by the Ethical Committee of Isfahan University of Medical sciences. IHHP was covered under IRB protocol FW A00008578. In this substudy we consider only elder population (over 65) with MetS.

2.2. Data Collection. Information on sociodemographic factors and self-reported medical history were obtained by interview. Anthropometric measurements, including height, weight, and waist and hip circumferences, were taken with subjects wearing light clothing by well-trained examiners. Waist circumference was measured to the nearest $0.1 \mathrm{~cm}$ in the horizontal plane at the high point of the iliac crest during minimal respiration [15]. Blood pressure was measured with a mercury sphygmomanometer using right arms, in a sitting position, after a $5 \mathrm{~min}$ rest. Systolic and diastolic blood pressure were recorded twice and averages were used for the data analysis. Blood samples were drawn from an antecubital vein after an $8-12 \mathrm{hr}$ overnight fast. Samples were stored at -20 until required for biochemical assays. Fasting venous blood samples were obtained from the antecubital vein between 08:00 and 09:30. Blood samples were centrifuged for $10 \mathrm{~min}$ at $906 \mathrm{~g}$ within $30 \mathrm{~min}$ of collection. Sera were analyzed for total cholesterol, high-density lipoprotein (HDL), triglycerides (TG), and fasting blood glucose (FBG). Lowdensity lipoprotein cholesterol (LDL) was calculated by the Friedewald equation when TG was less than $400 \mathrm{mg} / \mathrm{dL}$ [16].
TC was measured using enzymatic colorimetric methods. HDL was determined after dextran sulphate-magnesium chloride precipitation of HDL. All the tests were performed in the Central Laboratory of the Isfahan Cardiovascular Research Center and using autoanalyzer ELAN (Ependorf 2000). For quality control measures, this laboratory meets the criteria of the National Standard Laboratory (a WHO collaborating centre in Tehran).

2.3. MetS Definition. The ATPIII definition of MetS was met when three or more of the following criteria were present: waist circumference $\geq 102 \mathrm{~cm}$; HDL $<40 \mathrm{mg} / \mathrm{dL}$ or specific treatment for this lipid abnormality; triglycerides $\geq 150 \mathrm{mg} / \mathrm{dL}$ or specific treatment for this lipid abnormality; systolic blood pressure $\geq 130 \mathrm{mmHg}$ or diastolic blood pressure $\geq 85 \mathrm{mmHg}$ or treatment of previously diagnosed hypertension; fasting glucose $\geq 100 \mathrm{mg} / \mathrm{dL}$ [14].

2.4. Statistical Analysis. Data entry was carried out using EPI Info. Data were analyzed using STATA software (Stata/IC 11.0, StataCorp LP, College Station, TX, USA). For all analyses, statistical significance was assessed at the level of 0.05 (2tailed) and $P$ value $<0.05$ was considered as the borderline significance (marginal significance).

Receiver operating characteristic (ROC) analysis and the area under curve (AUC) were used to identify the sensitivity and specificity of anthropometric indices cut-off points for the detection of MetS without WC. The optimal cut-off values were defined as the point at which the value of "sensitivity + specificity - 1" was maximum (Youden's index). The Akaike information criterion (AIC) considered for comparing nonnested models and goodness of fit. Lower values of the index indicate the preferred model, that is, the one with the fewest parameters that still provides an adequate fit to the data.

\section{Results}

3.1. Baseline Characteristics. In total, 206 elderly subjects with metabolic syndrome were evaluated for this study. The mean age of participants was $71.85 \pm 5.44$. The mean of WC, BMI, WHR, and WHtR in the presence of MetS was $97.39 \pm$ $10.63,26.32 \pm 3.96,0.96 \pm 0.05,0.96 \pm 0.05$, and $58.55 \pm$ 6.11. Subjects with MetS had both higher systolic and diastolic blood pressure than subjects without MetS $(140.79 \pm 19.70$ and $81.84 \pm 4.39(P<0.001)$ versus $124.12 \pm 20.10$ and $77.41 \pm 10.78$ $(P<0.023))$ (Table 1$)$.

3.2. Obesity Indices and Metabolic Syndrome Except MetS Using ROC Curves. The predicting values for two or more metabolic risk factors and corresponding AUC of BMI, WC, WHR, and WHtR in men are shown in Table 2 and Figure 1. WC at a cut-off value of $94.5 \mathrm{~cm}$ resulted in the highest Youden index with corresponding sensitivity of $68 \%$ and $64 \%$ specificity to detect MetS. At a traditional cut-off value of $<102 \mathrm{~cm}$ of WC, the sensitivity dropped to $88 \%$, and specificity slightly rose to $23 \%$. 
TABLE 1: Characteristics of study subjects according to the presence of two or more metabolic risk factors except for WC in the elderly Iranians.

Men

Two or more metabolic risk factors of the NCEP-ATPIII of the criteria except for WC

\begin{tabular}{|c|c|c|c|c|}
\hline & Total & Absent & Present & $P$ value \\
\hline Age (years) & $71.85 \pm 5.44$ & $72.15 \pm 5.42$ & $71.71 \pm 5.49$ & 0.568 \\
\hline Body mass index $(\mathrm{kg} / \mathrm{m} 2)$ & $25.51 \pm 4.02$ & $24.36 \pm 3.83$ & $26.32 \pm 3.96$ & 0.001 \\
\hline WHR & $0.94 \pm 0.06$ & $0.93 \pm 0.06$ & $0.96 \pm 0.05$ & 0.001 \\
\hline Waist (cm) & $94.69 \pm 11.10$ & $90.86 \pm 10.66$ & $97.39 \pm 10.63$ & 0.000 \\
\hline WHtR & $56.91 \pm 6.51$ & $54.56 \pm 6.40$ & $58.55 \pm 6.11$ & 0.000 \\
\hline Fasting blood sugar (mg/dL) & $104.11 \pm 37.67$ & $90.12 \pm 18.53$ & $113.52 \pm 44.14$ & 0.000 \\
\hline Glucose (2hpp) (mg/dL) & $124.45 \pm 56.42$ & $103.48 \pm 28.21$ & $140.74 \pm 66.89$ & 0.000 \\
\hline Triglycerides (mg/dL) & $151.80 \pm 87.78$ & $101.25 \pm 33.41$ & $186.63 \pm 96.41$ & 0.000 \\
\hline HDL (mg/dL) & $40.74 \pm 10.64$ & $48.08 \pm 9.43$ & $35.80 \pm 8.31$ & 0.000 \\
\hline Cholesterol (mg/dL) & $199.56 \pm 39.40$ & $197.35 \pm 39.38$ & $200.68 \pm 39.43$ & 0.557 \\
\hline Systolic blood pressure & $134.12 \pm 21.40$ & $124.12 \pm 20.10$ & $140.79 \pm 19.70$ & 0.000 \\
\hline Diastolic blood pressure & $80.12 \pm 13.22$ & $77.41 \pm 10.78$ & $81.84 \pm 4.39$ & 0.023 \\
\hline Low HDL cholesterol $(n,(\%))$ & $98(48.5)$ & $13(15.9)$ & $85(70.8)$ & 0.000 \\
\hline High TG $(n,(\%))$ & $82(40.8)$ & $3(3.7)$ & $79(66.4)$ & 0.000 \\
\hline High blood pressure $(n,(\%))$ & $136(70.5)$ & $35(46.7)$ & $101(85.6)$ & 0.000 \\
\hline High blood sugar $(n,(\%))$ & $54(26.6)$ & $5(6.1)$ & $49(40.5)$ & 0.000 \\
\hline Lipid drug $(n,(\%))$ & $24(85.7)$ & $5(100.0)$ & $19(82.6)$ & 1.000 \\
\hline Diabetes drug regular $(n,(\%))$ & $30(96.8)$ & $5(100)$ & $25(96.2)$ & 1.000 \\
\hline Hypertension drug regular $(n,(\%))$ & $38(74.5)$ & $5(55.6)$ & $33(78.6)$ & 0.150 \\
\hline
\end{tabular}

The numerical values are presented as mean \pm SD and compared by Student's $t$-test except for items indicated by $₫$ that Mann-Whitney $U$ test was employed. Categorical data is shown as $n(\%)$ and tested by chi-square.

* BMI: body mass index, WC: waist circumference, WHR: waist-to-hip ratio, and WHtR: waist-to-height ratio.

${ }^{\dagger}$ Triglycerides $\geq 150 \mathrm{mg} / \mathrm{dL}$ or on antilipid agents.

${ }^{\dagger \dagger} \mathrm{HDL}-\mathrm{C}<40$ for men and $<50$ for women or on antilipid agents.

${ }^{\ddagger}$ High blood pressure is considered as $\mathrm{SBP} \geq 140 \mathrm{mmHg}$ or DBP $\geq 90 \mathrm{mmHg}$ or antihypertensive agents.

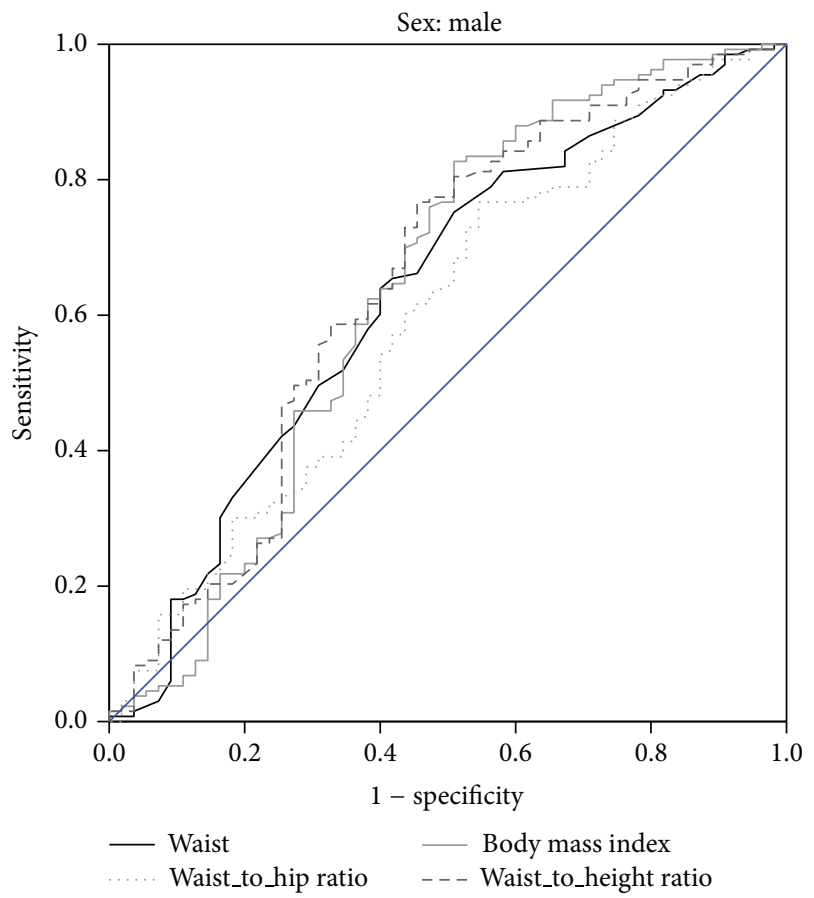

FIGURE 1: Receiver operating characteristics curve (ROC).
The BMI at a cut-off value of $\geq 28 \mathrm{~kg} / \mathrm{m}^{2}$ and the traditional cut-off value of $\geq 30 \mathrm{~kg} / \mathrm{m}^{2}$ were found to be having the lowest Youden index and corresponding sensitivity and specificity.

These results showed that WC (with the AUC of 0.590) was better indicator for high blood pressure compared to BMI and WHR, whereas BMI (with the AUC of 0.638) was better indicator of high blood pressure and WHR (with the AUC of 0.595 ) had a lower discriminating value. WC had greater AUC values compared to BMI and WHR in distinguishing low HDL cholesterol and hypertriglyceridemia only (0.609 and 0.625 , resp.). WHtR had better prediction ability to distinguish high blood pressure (AUC of 0.616 (0.527-0.705)).

Based on the indicator of interest, the optimum WC cutoff points in our study population for MetS were 94.5 and for MetS components ranged from 89.5 to 95.5 (Table 2). The best cut-off points of WHR for MetS were 0.95 and ranged from 0.92 to 0.97 for other MetS components. BMI at a cut-off value of the traditional cut-off value of $30 \mathrm{~kg} / \mathrm{m}^{2}$ were found to be having the lowest Youden index and corresponding sensitivity and specificity. Table 3 shows adjusted odds ratios (OR) for MetS. The model is adjusted for age and smoking status. The lowest AIC is related to WC in both adjusted and unadjusted model. So WC at a cut-off point of $94.5 \mathrm{~cm}$ 
TABLE 2: Areas under the ROC curve of WC, BMI, WHR, and WHtR to identify the presence of the metabolic risk factors other than WC in elderly men.

\begin{tabular}{|c|c|c|c|c|c|c|c|}
\hline & Obesity index & Best cut-off point & Sensitivity & Specificity & Youden & AUC (95\% CI) & $P$ value \\
\hline \multirow{4}{*}{ MetS } & WC & 94.5 & $64 \%$ & $68 \%$ & $32 \%$ & $0.683(0.606-0.761)$ & 0.000 \\
\hline & WHR & 0.95 & $6 \%$ & $69 \%$ & $29 \%$ & $0.645(0.563-0.727)$ & 0.001 \\
\hline & BMI & 26.65 & $48 \%$ & $76 \%$ & $24 \%$ & $0.641(0.561-0.722)$ & 0.001 \\
\hline & WHtR & 58.66 & $52 \%$ & 0.79 & $31 \%$ & $0.680(0.602-0.758)$ & \\
\hline \multirow{4}{*}{ High blood pressure } & WC & 89.5 & $75 \%$ & $49 \%$ & $24 \%$ & $0.633(0.542-0.724)$ & 0.004 \\
\hline & WHR & 0.92 & $76 \%$ & $45 \%$ & $22 \%$ & $0.595(0.503-0.687)$ & 0.040 \\
\hline & BMI & 22.84 & $82 \%$ & $49 \%$ & & $0.638(0.540-0.735)$ & 0.004 \\
\hline & WHtR & 53.84 & $76 \%$ & $54 \%$ & $31 \%$ & $0.616(0.527-0.705)$ & \\
\hline \multirow{4}{*}{ High triglyceride } & WC & 94.5 & $67 \%$ & $59 \%$ & $26 \%$ & $0.625(0.545-0.705)$ & 0.003 \\
\hline & WHR & 0.97 & $46 \%$ & $72 \%$ & $19 \%$ & $0.585(0.502-0.668)$ & 0.020 \\
\hline & BMI & 22.86 & $85 \%$ & $35 \%$ & $21 \%$ & $0.600(0.519-0.680)$ & 0.04 \\
\hline & WHtR & 53.09 & 0.85 & 0.38 & 0.22 & $0.607(0.527-0.687)$ & \\
\hline \multirow{4}{*}{ Low HDL } & WC & 94.5 & $63 \%$ & $58 \%$ & $21 \%$ & $0.609(0.527-0.690)$ & 0.010 \\
\hline & WHR & 0.96 & $52 \%$ & $68 \%$ & $20 \%$ & $0.597(0.516-0.679)$ & 0.010 \\
\hline & BMI & 24.83 & $63 \%$ & $52 \%$ & $15 \%$ & $0.579(0.498-0.661)$ & 0.060 \\
\hline & WHtR & 53.563 & $81 \%$ & $40 \%$ & $21 \%$ & $0.604(0.523-0.685)$ & \\
\hline \multirow{4}{*}{ High fasting blood sugar } & WC & 95.5 & $61 \%$ & $58 \%$ & $19 \%$ & $0.617(0.524-0.709)$ & 0.014 \\
\hline & WHR & 0.95 & $65 \%$ & $58 \%$ & $24 \%$ & $0.605(0.518-0.691)$ & 0.027 \\
\hline & BMI & 25.54 & $58 \%$ & $58 \%$ & $18 \%$ & $0.578(0.482-0.673)$ & 0.101 \\
\hline & WHtR & 56.04 & $98 \%$ & $15 \%$ & $13 \%$ & $0.512(0.431-0.593)$ & \\
\hline
\end{tabular}

BMI: body mass index, WC: waist circumference, WHR: waist-to-hip ratio, and WHtR: waist-to-height ratio.

TABLE 3: Association of the best cut-offs of obesity indices with MetS.

\begin{tabular}{|c|c|c|c|c|c|c|}
\hline & \multicolumn{3}{|c|}{ Crude OR (95\% CI) } & \multicolumn{3}{|c|}{ Adjusted OR (95\% CI) } \\
\hline & $\mathrm{OR}(\mathrm{CI})$ & $P$ value & AIC & $\mathrm{OR}(\mathrm{CI})$ & $P$ value & AIC \\
\hline WC & $3.835(0.00-7.11)$ & 0.000 & 237.55 & $4.564(2.37-8.81)$ & 0.000 & 240.19 \\
\hline WHR & $3.00(1.63-5.51)$ & 0.000 & 243.81 & $3.219(1.73-6.00)$ & 0.000 & 248.26 \\
\hline BMI & $2.954(1.55-5.62)$ & 0.001 & 247.1 & $3.115(1.62-5.98)$ & 0.001 & 251.78 \\
\hline WHtR & $4.023(2.07-7.80)$ & 0.000 & 238.121 & $4.162(2.13-8.12)$ & 0.000 & 243.09 \\
\hline
\end{tabular}

OR: odds ratio, CI: confidence interval, BMI: body mass index, WC: waist circumference, WHR: waist-to-hip ratio, and WHtR: waist-to-height ratio.

Model is adjusted for age and smoking status.

happened to be the best predictor of MetS. In the presented cut-off of WC the risk of MetS increased by 3.84.

\section{Discussion}

The findings of the present study demonstrated the superlative discriminating values of common anthropometric parameters for MetS in elderly Iranian men. While many studies were done among adolescents and adults, but to the best of our knowledge this is the first study which was done on elder men. After considering Youden's index, we found that the best cut-off points for WC in men are $94.5 \mathrm{~cm}$ instead of $102 \mathrm{~cm}$ which is recommended by ATPIII.

In recent years numerous studies have been done to find the best anthropometric indices for detecting MetS, especially, among different ethnics. In our previous study [9] we showed that, among the Iranian population, WC might be the most appropriate indicator to discriminate MetS regardless of gender and age, which has been confirmed with the present study. In this study we found that the best obesity indicator for distinguishing MetS among Iranian elderly men is WC.

There is controversy about the proper values of anthropometric values according to ethnicity, genetic background, sexes, and sociocultural aspects. Beydoun et al. showed that WC is among the most powerful tools to predict MetS and that optimal cut-off points for various indices including WC may differ by sex and race [17]. Beydoun's suggestion is consistent with other reports by Reeder et al. $[16,18]$ and Wang et al. [19]. While many studies have been done to find the best similar to our results. For example, Wakabayashi and Daimon illustrated that the associations between obesity and multiple risk factors for atherosclerosis become weaker as age increases, while age does not influence cut-off values of obesity indices except for higher WHtR at an older age in women [18]. Another study which was done by Shao et al. indicated that WHtR might be an optimal anthropometric predictor of MetS risk factors and the cut-off point of WHtR 
was approximately 0.50 in both genders of Chinese adults [20]. Likewise, another report by Dong et al. suggested that WHtR has better association with obesity related cardiovascular risk conditions in both sexes, except for hypertension in Chinese men [21]. An Iranian longitudinal study confirmed the cut-off points for women but decreased them to $90 \mathrm{~cm}$ for men. This might be in part due to the linear effect of WC on cardiovascular risk in men compared to women. Hence, among all eleven cut-off points, $90 \mathrm{~cm}$ was identified as the best definition of central obesity for men [10]. An eastern study which was done on Japanese subjects reported that VFA was better than WC and BMI for identification of subjects with two or more components of MetS [22, 23]. Another Japanese study has shown that VFA does not have better correlation with carotid intima-media thickness as a surrogate measurement of atherosclerosis than waist-hip ratio or WC [24]. A study among Korean adult male population reported WC cut-off point of $86.5 \mathrm{~cm}$ which is obtained cut points [25]. These major differences in the cut-off values might be attributed to ethnic variations and using different criteria for diagnosing MetS. Seo et al. claimed that because WC is clinically practical measurement and is more cost beneficial than direct imaging which is required for assessing visceral fat, so they suggested that WC measurements are sufficient for the detection of central obesity in correlation with the risk of MetS in elderly Koreans [25].

In this study we found that WC at a cut-off value of $94.5 \mathrm{~cm}$ has the highest sensitivity and specificity to predict the presence of 2 or more metabolic risk factors. When we applied the WC cut-off value of $\geq 102 \mathrm{~cm}$ for men and $\geq 88 \mathrm{~cm}$ for women as recommended by ATPIII criteria [14], the sensitivity to discriminate between those with and without MetS dropped from $64 \%$ to $68 \%$.

\section{Limitation}

The present study is limited by its cross-sectional nature, so we could not evaluate outcome measures. Consequently, the authors are mindful that differences could only be imputed from the previously documented data.

\section{Conclusion}

We found that BMI tended to be the weakest index for identifying MetS risk factors in elderly men. WC exhibited the best predictive index for MetS, almost similar to predictive powers of WHtR for identifying MetS. The two indices of WC and WHtR were better indicators of MetS. WC had WC had the highest sensitivity for MetS diagnosis among obesity indices. These cut-off values of WC should be advocated and used in Iranian elderly men.

\section{Conflict of Interests}

The authors declare that there is no conflict of interests regarding the publication of this paper.

\section{References}

[1] L. L. Hayman, J. C. Meininger, S. R. Daniels et al., "Primary prevention of cardiovascular disease in nursing practice: focus on children and youth: a scientific statement from the American Heart Association Committee on Atherosclerosis, Hypertension, and Obesity in Youth of the Council on Cardiovascular Disease in the Young, Council on Cardiovascular Nursing, Council on Epidemiology and Prevention, and Council on Nutrition, Physical Activity, and Metabolism," Circulation, vol. 116, no. 3, pp. 344-357, 2007.

[2] J. Steinberger and S. R. Daniels, "Obesity, insulin resistance, diabetes, and cardiovascular risk in children: an American Heart Association scientific statement from the Atherosclerosis, Hypertension, and Obesity in the Young Committee (Council on Cardiovascular Disease in the Young) and the Diabetes Committee (Council on Nutrition, Physical Activity, and Metabolism)," Circulation, vol. 107, no. 10, pp. 1448-1453, 2003.

[3] S. Y. Lee, H. S. Park, D. J. Kim et al., "Appropriate waist circumference cutoff points for central obesity in Korean adults," Diabetes Research and Clinical Practice, vol. 75, no. 1, pp. 72-80, 2007.

[4] H. Kaur, M. L. Hyder, and W. S. C. Poston, "Childhood Overweight: an Expanding Problem," Treatments in Endocrinology, vol. 2, no. 6, pp. 375-388, 2003.

[5] E. P. Whitlock, S. B. Williams, R. Gold, P. R. Smith, and S. A. Shipman, "Screening and interventions for childhood overweight: a summary of evidence for the US Preventive Services Task Force," Pediatrics, vol. 116, no. 1, pp. e125-e144, 2005.

[6] "Appropriate body-mass index for Asian populations and its implications for policy and intervention strategies," The Lancet, vol. 363, no. 9403, pp. 157-163, 2004.

[7] A. A. Shabnam, K. Homa, M. T. Reza, L. Bagher, F. M. Hossein, and A. Hamidreza, "Cut-off points of waist circumference and body mass index for detecting diabetes, hypercholesterolemia and hypertension according to National Non-Communicable Disease Risk Factors Surveillance in Iran," Archives of Medical Science, vol. 8, no. 4, pp. 614-621, 2012.

[8] R. Heshmat, P. Khashayar, H. R. A. Meybodi, M. R. Homami, and B. Larijani, "The appropriate waist circumference cut-off for Iranian population," Acta Medica Indonesiana, vol. 42, no. 4, pp. 209-215, 2010.

[9] M. Gharipour, N. Sarrafzadegan, M. Sadeghi et al., "Predictors of metabolic syndrome in the Iranian population: waist circumference, body mass index, or waist to hip ratio?" Cholesterol, vol. 2013, Article ID 198384, 6 pages, 2013.

[10] M. Talaei, N. G. Thomas, T. Marshall et al., "Appropriate cutoff values of waist circumference to predict cardiovascular outcomes: 7-year follow-up in an Iranian population," Internal Medicine, vol. 51, no. 2, pp. 139-146, 2012.

[11] T. Tchkonia, D. E. Morbeck, T. Von Zglinicki et al., "Fat tissue, aging, and cellular senescence," Aging Cell, vol. 9, no. 5, pp. 667684, 2010.

[12] J. C. Lovejoy, C. M. Champagne, L. De Jonge, H. Xie, and S. R. Smith, "Increased visceral fat and decreased energy expenditure during the menopausal transition," International Journal of Obesity, vol. 32, no. 6, pp. 949-958, 2008.

[13] M. Gharipour, M. Hosseini, E. Andalib, M. Bakhtiari Boroujeni, and N. Sarrafzadegan, "Effect of age on the phenotypeof metabolic syndrome in developing country," Advances in Biomedical Sciences. In press. 
[14] N. Sarraf-Zadegan, G. Sadri, H. Malek Afzali et al., "Isfahan Healthy Heart Programme: a comprehensive integrated community-based programme for cardiovascular disease prevention and control. Design, methods and initial experience," Acta Cardiologica, vol. 58, no. 4, pp. 309-320, 2003.

[15] M. Gharipour, R. Kelishadi, A. Khosravi, S. Shirani, M. Masjedi, and N. Sarrafzadegan, "The impact of a community trial on the pharmacological treatment in the individuals with the metabolic syndrome: findings from the Isfahan Healthy Heart Program, 2001-2007," Archives of Medical Science, vol. 8, no. 6, pp. 1009-1017, 2012.

[16] B. A. Reeder, A. Senthilselvan, J. P. Després et al., “The association of cardiovascular disease risk factors with abdominal obesity in Canada," Canadian Medical Association Journal, vol. 157, supplement 1, pp. S39-S45, 1997.

[17] M. A. Beydoun, M. T. Fanelli Kuczmarski, Y. Wang, M. A. Mason, M. K. Evans, and A. B. Zonderman, "Receiver-operating characteristics of adiposity for metabolic syndrome: the Healthy Aging in Neighborhoods of Diversity across the Life Span (HANDLS) study," Public Health Nutrition, vol. 14, no. 1, pp. 7792, 2011.

[18] I. Wakabayashi and T. Daimon, "Receiver-operated characteristics (ROCs) of the relationships between obesity indices and multiple risk factors (MRFs) for atherosclerosis at different ages in men and women," Archives of Gerontology and Geriatrics, vol. 55, no. 1, pp. 96-100, 2012.

[19] W. Wang, Y. Luo, Y. Liu et al., "Prevalence of metabolic syndrome and optimal waist circumference cut-off points for adults in Beijing," Diabetes Research and Clinical Practice, vol. 88, no. 2, pp. 209-216, 2010.

[20] J. Shao, L. Yu, X. Shen, D. Li, and K. Wang, "Waist-to-height ratio, an optimal predictor for obesity and metabolic syndrome in Chinese adults," Journal of Nutrition, Health and Aging, vol. 14, no. 9, pp. 782-785, 2010.

[21] X. Dong, Y. Liu, J. Yang, Y. Sun, and L. Chen, "Efficiency of anthropometric indicators of obesity for identifying cardiovascular risk factors in a Chinese population," Postgraduate Medical Journal, vol. 87, no. 1026, pp. 251-256, 2011.

[22] T. Hayashi, E. J. Boyko, M. J. McNeely, D. L. Leonetti, S. E. Kahn, and W. Y. Fujimoto, "Minimum waist and visceral fat values for identifying Japanese Americans at risk for the metabolic syndrome," Diabetes Care, vol. 30, no. 1, pp. 120-127, 2007.

[23] E. Oda, "Minimum waist and visceral fat values for identifying Japanese Americans at risk for the metabolic syndrome: response to Hayashi et al.," Diabetes Care, vol. 30, no. 5, article e40, 2007.

[24] R. Takami, N. Takeda, M. Hayashi et al., "Body fatness and fat distribution as predictors of metabolic abnormalities and early carotid atherosclerosis," Diabetes Care, vol. 24, no. 7, pp. 12481252, 2001.

[25] J. A. Seo, B. G. Kim, H. Cho et al., "The cutoff values of visceral fat area and waist circumference for identifying subjects at risk for metabolic syndrome in elderly Korean: Ansan Geriatric (AGE) cohort study," BMC Public Health, vol. 9, article 443, 2009. 


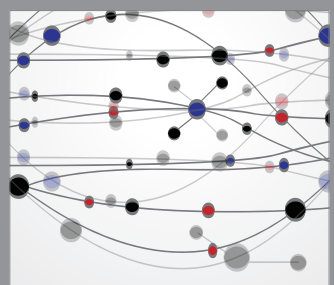

The Scientific World Journal
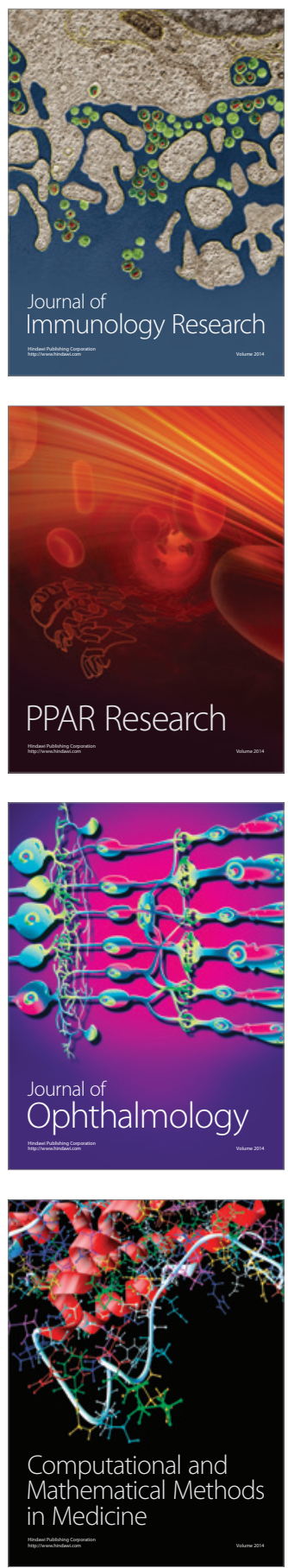

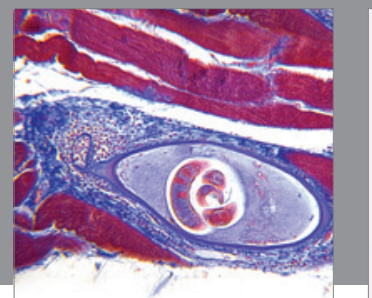

Gastroenterology

Research and Practice
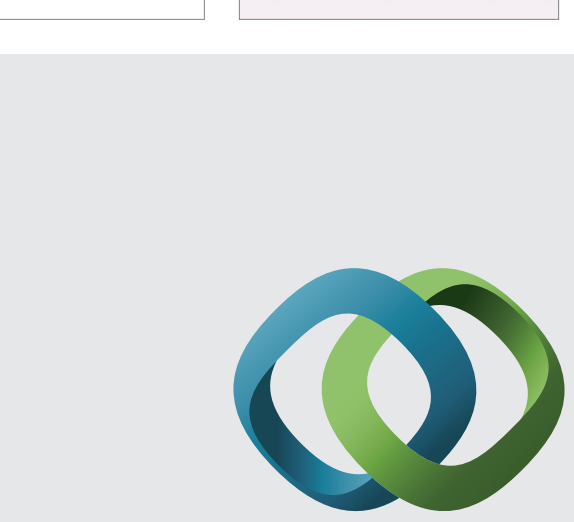

\section{Hindawi}

Submit your manuscripts at

http://www.hindawi.com
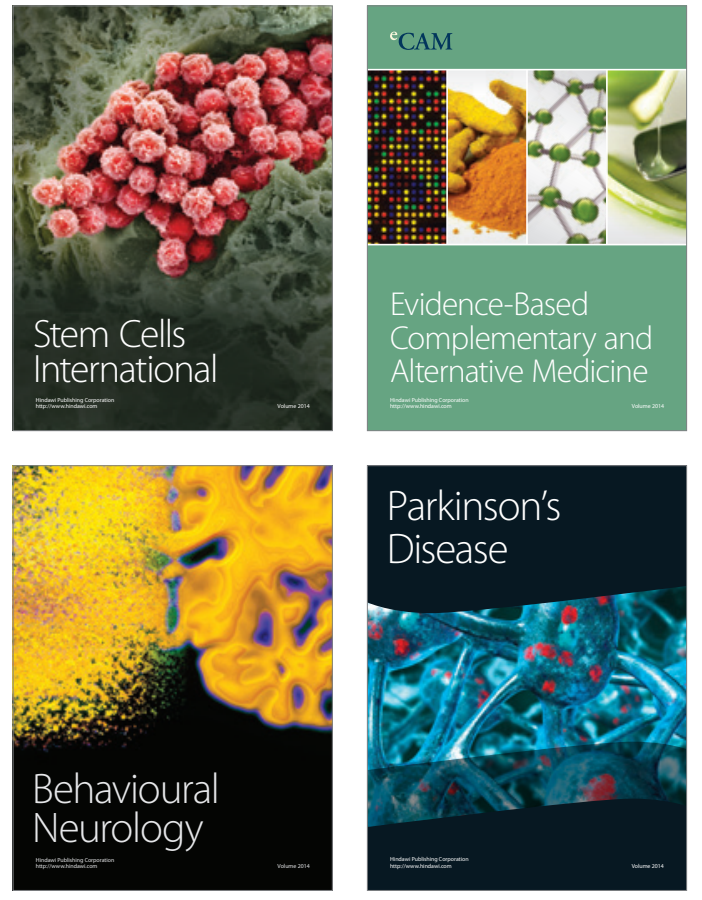
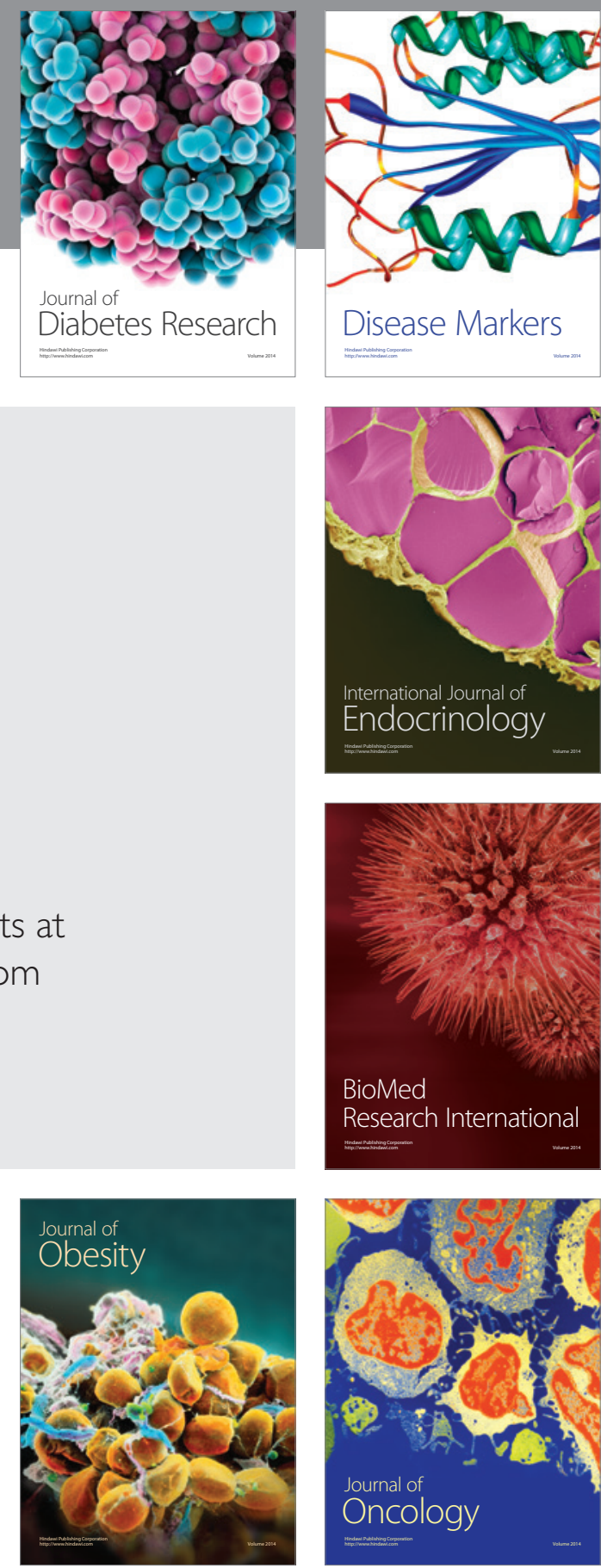

Disease Markers
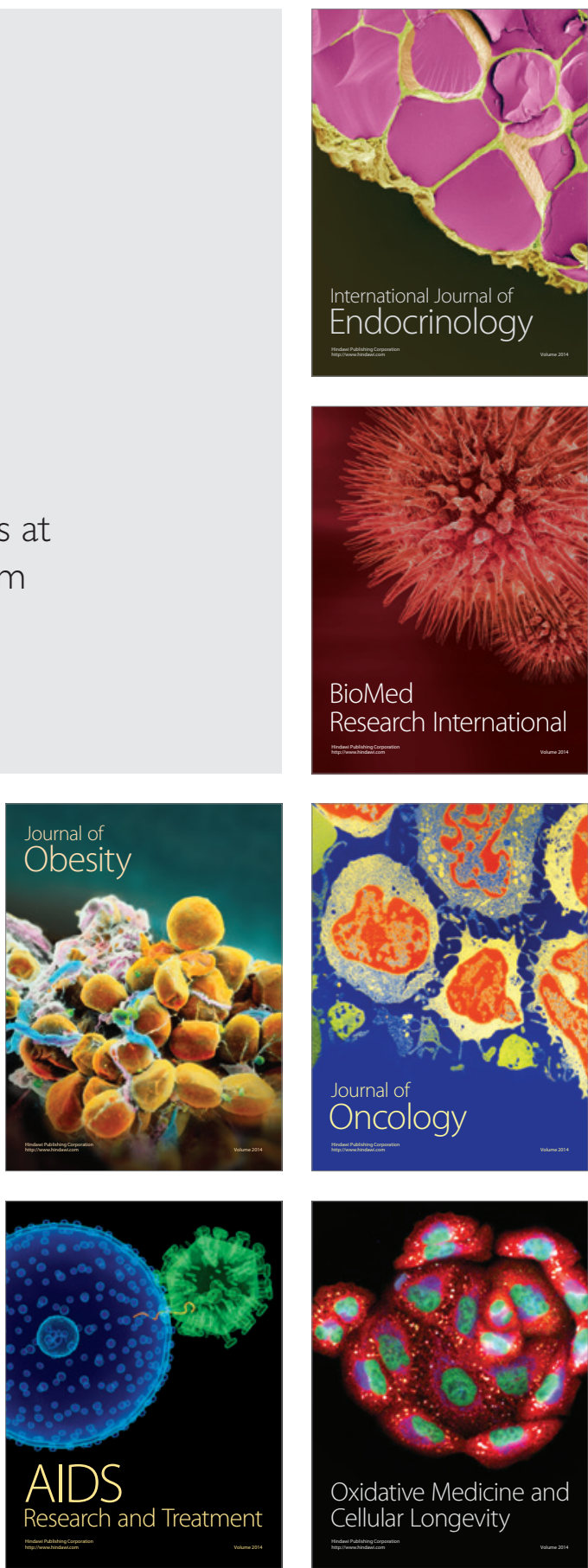